\title{
PATTERN AVOIDING PERMUTATIONS AND INDEPENDENT SETS IN GRAPHS
}

\author{
CHRISTIAN BEAN*, MURRAY TANNOCK ${ }^{\star}$, AND HENNING ULFARSSON*
}

\begin{abstract}
We introduce a new method for encoding permutations as weighted independent sets in a family of graphs we call cores. The encoding allows us to enumerate $(1324,2143)-,(1234,1324,2143)-,(1234,1324,1432,3214)$-avoiding permutations with respect to their number of "boundary points" and the size of the independent set in the graph they correspond to.
\end{abstract}

Keywords: permutation patterns, non-crossing subgraphs, independent sets

\section{INTRODUCTION}

The standardization of a string $s$ of distinct integers is the permutation st $(s)$ obtained by replacing the $i$ th smallest entry of $s$ with $i$. A permutation $\pi$ of length $n$ contains permutation $p$ of length $k$ if there is a subsequence (not necessarily consisting of consecutive entries) $\pi_{i_{1}} \pi_{i_{2}} \ldots \pi_{i_{k}}$ whose standardization is $p$. In this context, $p$ is called a (classical permutation) pattern. The subsequence in $\pi$ is called an occurrence of $p$. If no occurrence exists then $\pi$ avoids $p$. Take for example $\pi=51324$, which contains $p=123$ as the subsequences 134 and 124 , but avoids $p=231$.

Given a pattern $p$ we define $\operatorname{Av}_{n}(p)$ as the set of permutations of length $n$ that avoid $p$, and $\operatorname{Av}(p)=\cup_{n \geq 0} \operatorname{Av}_{n}(p)$. For a set $P$ of patterns we let $\operatorname{Av}_{n}(P)=\cap_{p \in P} \operatorname{Av}_{n}(p)$, and $\operatorname{Av}(P)=\cup_{n \geq 0} \operatorname{Av}_{n}(P)$. A permutation class is any set of permutations defined by the avoidance of a set of classical patterns.

We illustrate a connection between independent sets in graphs to certain pattern avoiding permutations. The classical problem of enumerating non-crossing subgraphs in a complete graph drawn on a regular polygon will turn out to be equivalent to a instance of 132-avoiding permutations. The methods developed can be extended to enumerate subclasses of permutations avoiding the pattern 1324, the only permutation class avoiding a single length 4 pattern that remains unenumerated.

\section{EnCoding Permutations as Weights on A GRID}

A letter $\pi_{i}$ in a permutation $\pi$ is called a left-to-right minimum if $\pi_{j}>\pi_{i}$ for all $j=1, \ldots, i-1$. The letter $\pi_{1}$ is always a left-to-right minimum in a non-empty permutation. Left-to-right maxima, right-to-left-minima and right-to-left-maxima are defined analogously.

The sequence of left-to-right minima of a permutation $\pi$ will be called the $\mathrm{lrm}$ boundary of the permutation and denoted $\operatorname{lrm}(\pi)$, e.g., if $\pi=845367912$ then $\operatorname{lrm}(\pi)=$

2010 Mathematics Subject Classification. Primary: 05A05; Secondary: 05A15.

* Research partially supported by grant 141761-051 from the Icelandic Research Fund. 
8431. Given any permutation with $n$ left-to-right-minima, we can arrange the lrmboundary on a northwest-southeast diagonal and insert the remaining points of the permutation in a staircase (grid), $B_{n}$, above this diagonal. This grid is a collection of boxes with integer coordinates, labeled in matrix notation, so the the top row has cells $(11),(12), \ldots,(1 n)$, from left to right, the next row has cells $(22),(23), \ldots,(2 n)$, and so on. Note that the lrm-boundary is a part of the grid. See Figure (1b) for an example with the permutation 845367912 .

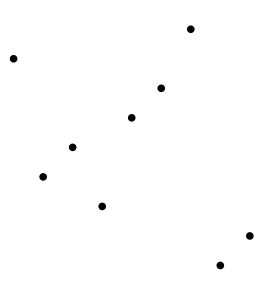

(a) The plot of the points $\left(i, \pi_{i}\right)$ of the permutation on the Cartesian plane.

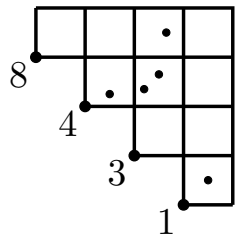

(b) The permutation drawn on a staircase, $B_{4}$. The lrmboundary is drawn on a diagonal.

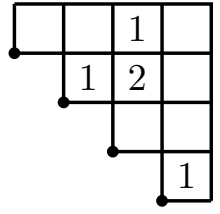

(c) The permutation encoded as the number of points in each box in the staircase.

FIGURE 1. Three representations of the permutation $\pi=845367912$.

We can give a coarser representation of this permutation by recording the number of points in each box of the grid, see Figure (1c). This is the (staircase) encoding of the permutation, denoted enc $(\pi)$. It is not necessary to label the left-to-right minima, their values can be inferred from the number of points in each box. There are other permutations (of length 8) that have the encoding in Figure (1c), e.g., 846379512.

For permutations avoiding 132, the presence of points in a box may constrain other boxes to be empty. For example, in the encoding in Figure (1c), the third box in row 1 is occupied, which implies the right-most boxes in rows 2 and 3 must be empty. These constraints are symmetric. To capture these constraints we create a graph by placing a vertex for every box and an edge between boxes that exclude one another. More formally:

Definition 2.1. Let $n \geq 0$ be an integer. The 132-core of size $n$ is the labeled, undirected graph $D_{n}$ with vertex set $\{(i j): i=1, \ldots, n, j=i, \ldots, n\}$ and an edge between $(i j)$ and $(k \ell)$ if $i<k, j<\ell$ and the rectangle $\{i, i+1, \ldots, k\} \times\{j, j+1, \ldots, \ell\}$ is a subset of the vertex set.

If $n=0$, the 132-core is the empty graph. See Figure2 (left) for the set of neighbours of a cell in a 132-core, and Figure 3 for the 132-cores of sizes $n=1, \ldots, 5$. We use the letter " $D$ " in anticipation of Definition 4.3. Until we define another type of core in Section 3 we will refer to 132-cores as cores.

Lemma 2.2. The staircase encoding, restricted to the set of permutation avoiding 132 with exactly $n$ left-to-right minima, is a bijection to the set of weighted independent set of $D_{n}$.

Proof. A rectangular region in a 132-avoiding permutation drawn on a staircase grid (as in Figure $(1 \mathrm{~b})$ ) is increasing. Therefore, from the definition of the 132-cores, the 

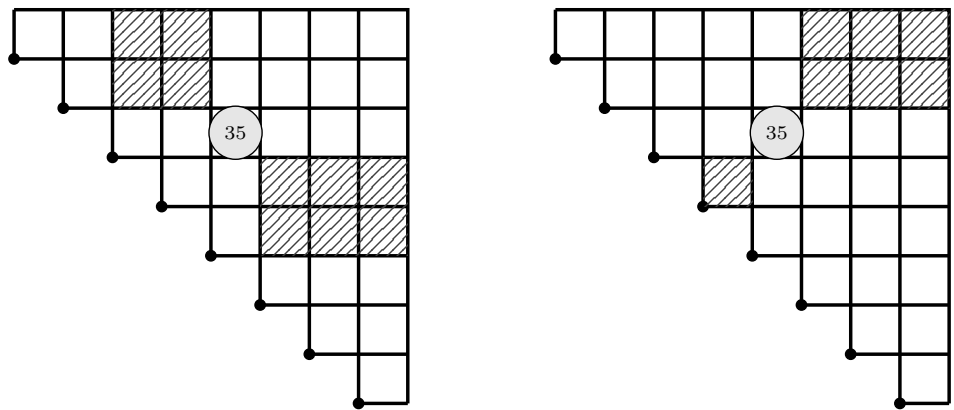

Figure 2. The cell (35) with its set of neighbours shaded; on the left in the 132-core (Definition 2.1), and on the right in the 123-core (Definition 3.1).

staircase encoding, restricted to the set of 132 -avoiding permutations with $n$ left-to-right minima, is a bijection to the set of weighted independent sets of $D_{n}$.

For a concrete example, consider the permutation 845367912 from Figure (1b). We can encode it as the independent set $\{(13),(22),(23),(44)\}$ in $D_{4}$ along with the sequence of positive integer weights $(1,1,2,1)$ recording the number of points at each vertex in the independent set.

From the definition, it is easy to see that $D_{n}$ has $1+2+\cdots+n=\left(\begin{array}{c}n+1 \\ 2\end{array}\right)$ vertices. To compute the number of edges consider a vertex $(1 j)$ in the first row of $D_{n}$. It has neighbours in the rectangular region $\{2, \ldots, j\} \times\{j+1, \ldots, n\}$, giving a total of $(j-1)(n-j)=(n+1) j-j^{2}-n$ edges. The first row therefore contributes

$$
(n+1)\left(\begin{array}{c}
n+1 \\
2
\end{array}\right)-\frac{2 n+1}{3}\left(\begin{array}{c}
n+1 \\
2
\end{array}\right)-n^{2}=\frac{n+2}{3}\left(\begin{array}{c}
n+1 \\
2
\end{array}\right)-n^{2}=\left(\begin{array}{l}
n \\
3
\end{array}\right)
$$

edges. If we only consider edges going to the southeast then row $i$ in the core of size $n$ will look precisely like the top row in a smaller core of size $n-i+1$. The total number of edges in $D_{n}$ is therefore $\left(\begin{array}{c}n+1 \\ 4\end{array}\right)$. The enumerations of vertices and edges in the cores are special cases of the following result.

Proposition 2.3. The number of cliques of size $k$ in $D_{n}$ is $\left(\begin{array}{c}n+1 \\ 2 k\end{array}\right)$.

Proof. The case of $k=1$ (vertices) and $k=2$ are given above. A clique of size $k>2$ in $D_{n}$ either has:
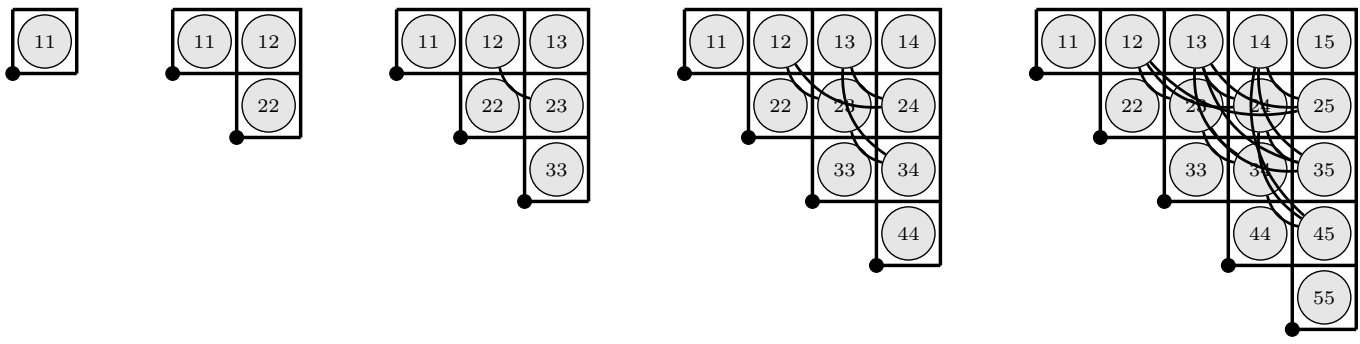

FiguRE 3 . The 132-cores of sizes $1, \ldots, 5$. 
- no vertex in the first row of the core, or

- has exactly one vertex in the first row.

In the first case, we have $\left(\begin{array}{c}n \\ 2 k\end{array}\right)$ cliques of size $k$, by induction. In the second case, assume the vertex in the first row is $(1 j)$. The remaining vertices in the clique must form a clique of size $k-1$ in the rectangular region $\{2, \ldots, j\} \times\{j+1, \ldots, n\}$. Such a clique is obtained by choosing rows $r_{1}<r_{2}<\cdots<r_{k-1}$, and independently columns $c_{1}<c_{2}<$ $\cdots<c_{k-1}$. Such a choice corresponds to the clique $\left(r_{1}, c_{1}\right),\left(r_{2}, c_{2}\right), \ldots,\left(r_{k-1}, c_{k-1}\right)$. The number of these choices is $\left(\begin{array}{l}j-1 \\ k-1\end{array}\right)\left(\begin{array}{l}n-j \\ k-1\end{array}\right)$. Thus the number of cliques is

$$
\left(\begin{array}{c}
n \\
2 k
\end{array}\right)+\sum_{j=k}^{n-k+1}\left(\begin{array}{l}
j-1 \\
k-1
\end{array}\right)\left(\begin{array}{l}
n-j \\
k-1
\end{array}\right) .
$$

The sum is easily seen to be $\left(\begin{array}{c}n \\ 2 k-1\end{array}\right)$, which completes the proof.

Because of Lemma 2.2 the number of independent sets in the core is more relevant for our purposes. In the following theorem we use a multivariate generating function to enumerate the number of independent sets of a given size. The variable $x$ tracks the number of boundary points (which are left-to-right minima until we consider other boundaries) and $y$ tracks the size of the independent set. When we modify the generating function to enumerate permutations we let $z$ track the number of points in the permutation and $t$ track the number of boundary points.

Theorem 2.4. The number of independent sets of size $k$ in the 132-core of size $n$ is given by the coefficient of $x^{n} y^{k}$ in the generating function $F=F(x, y)$ satisfying the functional equation

$$
F=1+x F+\frac{x y F^{2}}{1-y(F-1)}
$$

Solving this equation gives,

$$
F=\frac{(1-x)(1+y)+y-\sqrt{x(1+y)(x y+x-4 y-2)+1}}{2 y} .
$$

Proof. If $n=0$ the core is empty and the empty set is the only independent set. This gives the term $x^{0} y^{0}=1$ in Equation (11) below. If $n>0$ then we consider two cases: First, the case when the independent set has no vertex in the top row of the core. This type of independent set corresponds to an independent set in the core of size $n-1$ which is isomorphic to the subgraph induced by the vertices in rows $2, \ldots, n$. This gives the term $x F$ in the equation below. Finally, we consider the case when the independent set has vertices in the top row. There are no edges between vertices in the top row, therefore any subset of vertices $\left\{1 x_{1}, 1 x_{2}, \ldots, 1 x_{d}\right\}$ (with $x_{i}<x_{j}$ if $i<j$ ) can be in the independent set. The neighbours of $\left(1 x_{1}\right)$ form a rectangular region $\left\{2, \ldots, x_{1}\right\} \times\left\{x_{1}+1, \ldots, n\right\}$ in rows $2, \ldots, x_{1}$. The remaining vertices in these rows form a core of size $x_{1}-1$. This can be visualized on the staircase grid, as in Figure 4. Similarly, for $1<i \leq d$, the neighbours of $\left(1 x_{i}\right)$ form a rectangular region $\left\{2, \ldots, x_{i}\right\} \times\left\{x_{i}+1, \ldots, n\right\}$ in rows $2, \ldots, x_{i}$. The remaining vertices in rows $x_{i-1}+1, \ldots, x_{i}$ form a core of size $x_{i}-x_{i-1}$. Lastly, the cells in rows $x_{d+1}, \ldots, n$ do 
not share an edge with a vertex in the top row, and form a core of size $n-d$. This case gives the rest of the terms in the equation below.

$$
\begin{aligned}
F & =1+x F+x y F^{2}+\cdots+x y^{n} F^{2}(F-1)^{n-1}+\cdots \\
& =1+x F+\frac{x y F^{2}}{1-y(F-1)} .
\end{aligned}
$$

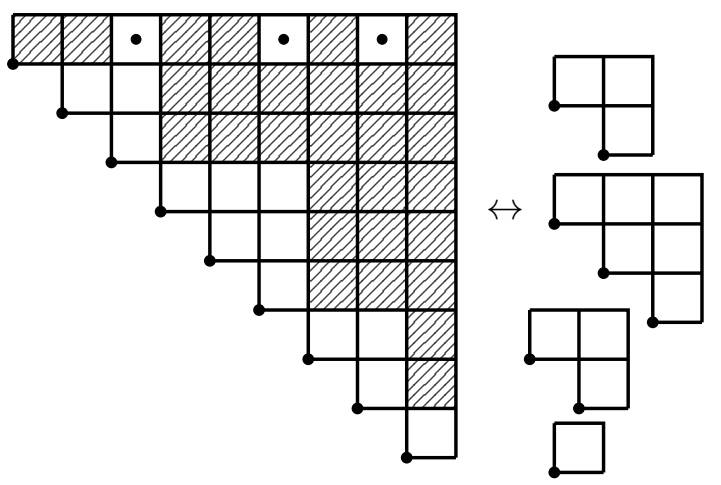

Figure 4 . The staircase grid $D_{9}$, where vertices (13), (16), (18) are in the independent set. The vertices which cannot be added to make an independent set are shaded. The induced subgraphs are shown on the right.

To obtain a formula for the coefficients of the generating function $F(x, y)$ we can make the substitution $F=1+w$ in the functional equation in Theorem 2.4 Rearranging produces the equation.

$$
w=x(1+y)\left(\frac{1+w}{1-y w}\right) \text {. }
$$

This same equation appears in Section 2.2 in Flajolet and Noy [6] where they enumerate non-crossing subgraphs of a complete graph drawn on a polygon. Like there we can apply Lagrange inversion (see e.g., Comtet [5]) to obtain the coefficients:

Corollary 2.5 (Theorem 2 (ii) in Flajolet and Noy [6]). The number of independent sets of size $k$ in the 132-core of size $n$ is given by

$$
I(n, k)=\frac{1}{n} \sum_{j=0}^{n-1}\left(\begin{array}{c}
n \\
k-j
\end{array}\right)\left(\begin{array}{c}
n \\
j+1
\end{array}\right)\left(\begin{array}{c}
n-1+j \\
n-1
\end{array}\right) .
$$

Setting $y=z /(1-z)$, to allow an arbitrarily long (non-empty) increasing sequence instead of a vertex in an independent set, and $x=t z$, to track the number of left-to-right minima, in the generating function in Theorem 2.4 gives the generating function

$$
\frac{z-t z+1-\sqrt{(t-1)^{2} z^{2}-2(t+1)+1}}{2 z}
$$

This is the well-known generating function for the Narayana numbers [13, A001263] enumerating the permutations avoiding 132 by their number of left-to-right minima. 
These numbers are usually arranged in a triangle, whose $n$-th row (starting from 0 ) contains the number of 132-avoiding permutations of length $n$ with $k$ left-to-right minima (the coefficient of $z^{n} t^{k}$ ). The triangle is often called a Catalan-triangle since the row sums are Catalan numbers, see Table 1 .

$\begin{array}{lllllllllllll}1 & & & & & & & & & \\ 1 & & & & & & & & & \\ 1 & 1 & & & & & & & & \\ 1 & 3 & 1 & & & & & & & \\ 1 & 6 & 6 & 1 & & & & & & \\ 1 & 10 & 20 & 10 & 1 & & & & & \\ 1 & 15 & 50 & 50 & 15 & 1 & & & & & \\ 1 & 21 & 105 & 175 & 105 & 21 & 1 & & & & \\ 1 & 28 & 196 & 490 & 490 & 196 & 28 & 1 & & & \\ 1 & 36 & 336 & 1176 & 1764 & 1176 & 336 & 36 & 1 & & \\ 1 & 45 & 540 & 2520 & 5292 & 5292 & 2520 & 540 & 45 & 1 & \\ 1 & 55 & 825 & 4950 & 13860 & 19404 & 13860 & 4950 & 825 & 55 & 1\end{array}$

TABLE 1. The Catalan triangle of Narayana numbers [13, A001263].

Setting $t=1$ in (2), and thus forgetting the information about the left-to-right minima, gives the usual generating function of the Catalan numbers.

Instead of enumerating $\operatorname{Av}(132)$ by the number of left-to-right minima we can enumerate them by the size of the independent set in the corresponding core.

Proposition 2.6. The number of permutations avoiding 132 of length $\ell$ produced by inflating an independent set of size $k$ is

$$
\sum_{n=0}^{\ell} I(n, k)\left(\begin{array}{c}
\ell-n-1 \\
k-1
\end{array}\right)
$$

where $I(n, k)$ is defined in Corollary 2.5.

Proof. The sum is taken over the number of left-to-right minima and the binomial coefficient counts the number of compositions of the remaining $\ell-n$ points into $k$ non-empty parts, corresponding to the vertices of the independent set.

The proposition gives a new Catalan triangle, shown in Table 2, which has been added to the Online Encyclopedia of Integer Sequences [13, A262370].

In the triangle, the right-most numbers in rows $2,5,8,11,14$ form the sequence

$$
1,1,2,5,14 \text {, }
$$

and the right-most numbers in rows $1,4,7,10,13$ form the sequence

$$
1,3,9,30,105 \text {. }
$$

This leads to the following conjecture, where the first 8 terms of the sequences have been checked.

Conjecture 2.7. (1) The right-most numbers in rows numbered $2+3 i, i=0,1 \ldots$ are the Catalan numbers. 
PATTERN AVOIDING PERMUTATIONS AND INDEPENDENT SETS

1

11

14

$1 \quad 10 \quad 3$

$1 \quad 20 \quad 20 \quad 1$

$\begin{array}{llll}1 & 35 & 77 & 19\end{array}$

$\begin{array}{lllll}1 & 56 & 224 & 139 & 9\end{array}$

$\begin{array}{llllll}1 & 84 & 546 & 656 & 141 & 2\end{array}$

$1 \quad 120 \quad 1176 \quad 2375 \quad 1104 \quad 86$

$\begin{array}{lllllll}1 & 165 & 2310 & 7172 & 5937 & 1181 & 30\end{array}$

$\begin{array}{llllllll}1 & 220 & 4224 & 18953 & 24959 & 9594 & 830 & 5\end{array}$

$\begin{array}{llllllll}1 & 286 & 7293 & 45188 & 87893 & 56358 & 10613 & 380\end{array}$

$\begin{array}{lllllllll}1 & 364 & 12012 & 99242 & 270452 & 264012 & 88472 & 8240 & 105\end{array}$

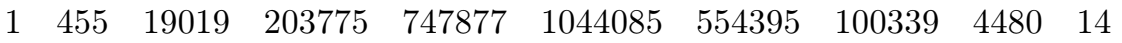

TABle 2. The Catalan triangle given by Proposition 2.6.

(2) The right-most numbers in rows numbered $1+3 i, i=0,1 \ldots$ are the central elements of the (1,2)-Pascal triangle [13, A029651].

The reader might wonder about the sequence of right-most numbers in the remaining rows. We have not found a particular structure in that sequence, and it does not appear in the Online Encyclopaedia of Integer Sequences [13].

\section{Permutations AVoiding 123}

Consider drawing a permutation that avoids 123 on a staircase grid. Take for example the permutation 639871542 shown in Figure 5. Every rectangular region of boxes is

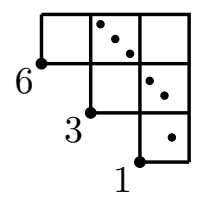

Figure 5. The permutation 639871542 drawn on a staircase grid. The left-to-right minima, 6,3 and 1 , are drawn on a diagonal.

decreasing, instead of increasing in the case of the 132-avoiding permutations, and the existence of points in a box implies boxes southwest and northeast of it must be empty. We are naturally lead to the following definition:

Definition 3.1. Let $n \geq 0$ be an integer. The 123-core of size $n$ is the labeled, undirected graph $U_{n}$ with vertex set $\{(i j): i=1, \ldots, n, j=i, \ldots, n\}$ and an edge between $(i j)$ and $(k \ell)$ if $i>k, j<\ell$ and the rectangle $\{k, k+1, \ldots, i\} \times\{j, j+1, \ldots, \ell\}$ is a subset of the vertex set.

See Figure 2 (right) for the set of neighbours of a cell in a 132-core, and Figure 6 for the 123-cores of sizes $n=1, \ldots, 5$. We use the letter " $U$ " in anticipation of Definition 4.3 . 

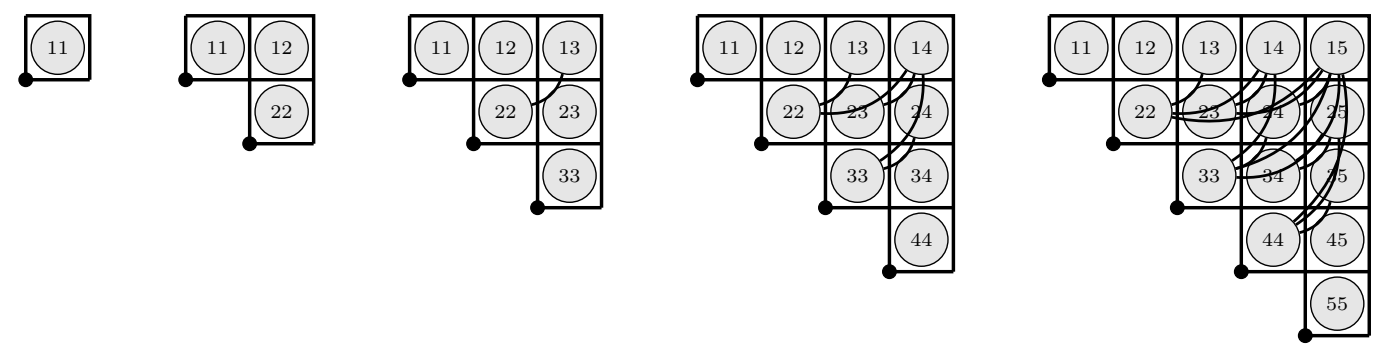

Figure 6. The 123-cores of sizes $1, \ldots, 5$.

The 123-core $U_{n}$ is isomorphic to $D_{n}$ (the 132-core of size $n$ ) when $n=0,1,2,3$. However, $D_{4}$ is a 5 -cycle, whereas $U_{4}$ is not.

Even though the 123-cores are not isomorphic to the 132-cores in general they have the same number of cliques of each size. This can be proven with the same method as Proposition 2.3

Proposition 3.2. The number of cliques of size $k$ in $U_{n}$ is $\left(\begin{array}{c}n+1 \\ 2 k\end{array}\right)$.

Moreover, it turns out that the number of independent sets of each size is the same in 123-cores as in 132-cores.

Theorem 3.3. The number of independent sets of size $k$ in the 123-core of size $n$ is given by the coefficient of $x^{n} y^{k}$ in the generating function $F=F(x, y)$ satisfying the functional equation

$$
F=1+x F+\frac{x y F^{2}}{1-y(F-1)} .
$$

We leave it to the reader to prove this with the method used to prove Theorem 2.4 with the modification that we consider the number of non-empty boxes on the diagonal instead of the top row, see Figure 7.
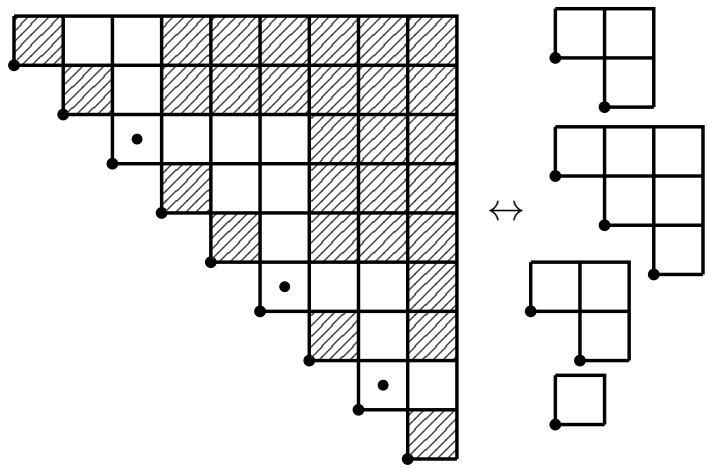

FiguRE 7. Illustration of how to prove Theorem 3.3 using a modification of the method in the proof of Theorem 2.4.

Theorem 3.3 implies that the bivariate generating function in Theorem (2.4), the enumerations in Proposition 2.6 and Corollary 2.5. and the Catalan triangles in Tables 1 and 2 also apply to permutations avoiding 123. 


\section{Permutations avoiding 1324}

In this section we generalize the cores defined above with the goal of enumerating subclasses of $\operatorname{Av}(1324)$. We start with the theory of these new cores. The first subclass considered is $\operatorname{Av}(1324,2143)$ in subsection 4.1. In the following two subsections we enumerate $\operatorname{Av}(1234,1324,2143)$ and $\operatorname{Av}(1234,1324,1432,3214)$. The method of proof for all of these classes is to consider the subsequence of points in a permutation containing the left-to-right minima and the right-to-left maxima. We then find a structural description of these boundaries, and use the generalized cores to find the enumeration of the original class. The reason to focus on subclasses of the permutation class $\operatorname{Av}(1324)$, is because it is the only unenumerated class avoiding a single length 4 pattern. The theory we develop also applies to subclasses of $\operatorname{Av}(1234)$, which has a known enumeration, first found by Gessel [8], and subclasses of $\operatorname{Av}(1234,1324)$, which is conjectured to be enumerated by a non- $D$-finite generating function, see Albert et al. [1, Section 6.2].

For these classes of permutations, the staircase encoding defined above is no longer unique, the simplest example being the permutations 123 and 132 which belong to all three classes and have the same staircase encoding. To remedy this we consider the right-to-left maxima (rlm) of a permutation:

Definition 4.1. Given a permutation $\pi$, we define its boundary, $\partial(\pi)$, as the standardization of the subsequence of $\pi$ containing the left-to-right minima and the right-to-left maxima.

For example if $\pi=(11)(12)(13)(10) 293671845$, as in Figure 8 , then $\partial(\pi)$ is the standardization of $(11)(13)(10) 29185$, i.e., 87625143. By construction $\partial(\pi)$ avoids 123 , and every permutation that avoids 123 is its own boundary.

Definition 4.2. Given a permutation $\pi$ that avoids 123 we define its boundary grid, $\operatorname{bg}(\pi)$, as: (1) the collection of 1-by-1 boxes whose corners have integer coordinates, with the requirement that the lower left corner of each box is northeast of a left-toright minimum, and the upper right corner of each box is southwest of a right-to-left maximum; and (2) the collection of points which are either a left-to-right minimum, or a right-to-left maximum.

For example, the boundary grid of 87625143 is the boundary grid in Figure 8 , For a permutation $\pi$ we create its (boundary) encoding, benc $(\pi)$, by writing the number of points in each box of the boundary grid of $\partial(\pi)$; see Figure 8 (right).

We generalize the construction of the 132- and 123-cores above:

Definition 4.3. A grid is a collection of 1-by-1 boxes whose corners have integer coordinates, along with a collection of points with integer coordinates, such that no two points lie on the same horizontal or vertical line 1 Given such a grid $B$ we define the

(1) down-core as the graph $D(B)$ whose vertices are the boxes in the grid and an edge between boxes $(i j),(k \ell)$ if $i<k, j<\ell$ and the rectangle $\{i, i+1, \ldots, k\} \times$ $\{j, j+1, \ldots, \ell\}$ is a subset of $B$.

\footnotetext{
${ }^{1}$ For consistency with the staircase grids we use matrix notation here as well. We use the convention that the left-most box, or point, of a grid has $x$ coordinate 1, and the top-most box, or point, of a grid has $y$ coordinate 1 .
} 


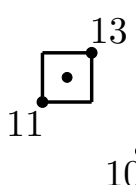

10

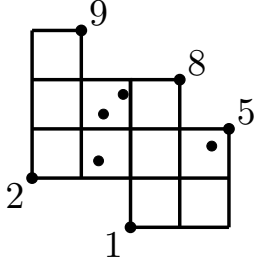

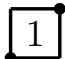

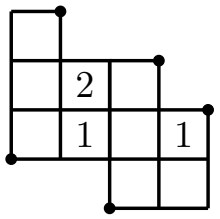

Figure 8. On the left, the permutation (11)(12)(13)(10)293671845 is drawn on a boundary grid highlighting its lrm's and rlm's. On the right, we have the boundary encoding of the same permutation.

(2) up-core as the graph $U(B)$ whose vertices are the boxes in the grid and an edge between boxes $(i j),(k \ell)$ if $i>k, j<\ell$ and the rectangle $\{k, k+1, \ldots, i\} \times$ $\{j, j+1, \ldots, \ell\}$ is a subset of $B$.

(3) updown-core as the graph $U D(B)$ whose vertices are the boxes in the grid and an edge between boxes $(i j),(k \ell)$ if one of the following conditions is satisfied: (a) There is an edge between $(i j),(k \ell)$ in $D(B)$; (b) there is an edge between $(i j),(k \ell)$ in $U(B)$; (c) if $i=k, \ell<j$ and rectangle $\{i\} \times\{\ell, \ell+1, \ldots, j\}$ is a subset of $B$; (d) if $i<k, j=\ell$ and rectangle $\{i, i+1, \ldots, k\} \times\{j\}$ is a subset of $B$.

See Figure 9 for an example of the different types of cores.
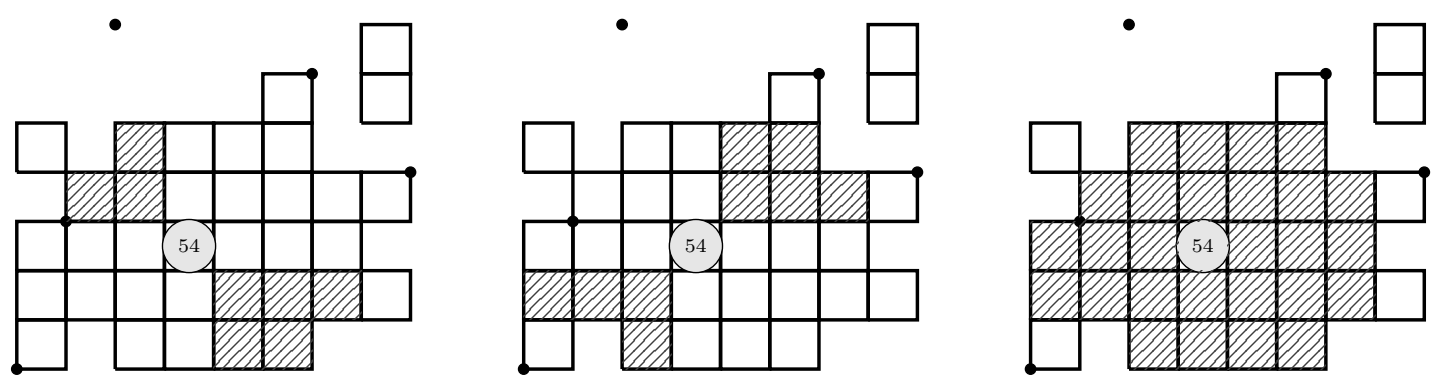

Figure 9. The cell (54) with its set of neighbours shaded; on the left in the down-core, in the middle in the up-core, and on the right in the updown-core.

We note that the 132-core is the down-core for the staircase grid while the 123-core is the up-core for the same grid.

For two permutations $\sigma$ and $\tau$ the skew-sum, $\sigma \ominus \tau$, is the permutation created by concatenating $\sigma$ and $\tau$ after adding the length of $\tau$ to each element of $\sigma$. For example, $1432 \ominus 3142=58763142$. A permutation is skew-decomposable if it can be written as a non-trivial skew-sum. By convention, the empty permutation is skew-decomposable. 
Lemma 4.4. A 123-avoiding permutation $\pi$ of length greater than 1 is skew-indecomposable if and only if $U D(\mathrm{bg}(\pi))$ is connected and every left-to-right minimum is attached to some 1-by-1 box in $\operatorname{bg}(\pi)$.

Proof. Assume $\pi$ is skew-decomposable as $\pi=\sigma \ominus \tau$ where $\sigma$ has length $s$, and $\tau$ is skew-indecomposable. If $\tau=1$ then it is a left-to-right minimum not attached to a box. Otherwise there is at least one box in the boundary grid of $\tau$. Either $\sigma$ has a boundary grid consisting of just integer points, in which case we are done; or it has at least one box which is disconnected from the box in $\tau$.

For the other direction, if there is a left-to-right minimum that is not attached to a box then it is a component in the skew-decomposition of $\pi$. Otherwise there must be exist two distinct subsets, $B_{1}$, and $B_{2}$, of boxes which are connected components. We can assume that all the boxes in $B_{1}$ are to the left and above all the boxes in $B_{2}$. The left-to-right minima, and the right-to-left maxima, attached to boxes in $B_{1}$ must be to the left and above the corresponding points attached to boxes in $B_{2}$. This implies that the points attached to $B_{1}$ are in a different component than the points attached to $B_{2}$ are, in the skew-decomposition of $\pi$.

For the subclasses considered below we will begin with finding the generating function for the skew-indecomposable permutations in the class. We will need rely on the following easily proven remark to translate such a generating function to the entire class.

Remark 4.5. If a permutation class $\mathcal{C}$ has basis $P$ and the patterns in $P$ are skewindecomposable then the skew-sum of two permutations from $\mathcal{C}$ is another permutation in $\mathcal{C}$. For such classes the generating functions enumerating the class, $C$, and the skew-indecomposable permutations in the class, $C_{i n d}$ are related by the formula

$$
C=\frac{1}{1-C_{\text {ind }}}
$$

because every permutation in the class has a unique decomposition into skew-indecomposable permutations. This property holds for the classes studied in this section.

Definition 4.6. Let $B$ be the boundary grid of a permutation $\pi$ avoiding 123 .

(1) Let dperms $(B)$ be the set of permutations obtained by choosing a weighted independent set in the down-core $D(B)$ and inflating the vertices of the independent set into a increasing sequence of points, whose length is determined by the weight of the vertex, while making sure every rectangular region inside the grid is increasing.

(2) Likewise, we define uperms $(B)$ using the up-core, by inflating weights into decreasing sequences.

(3) Finally we define udperms $(B)$ using the updown-core, by inflating an (unweighted) independent set into a single point for each vertex.

For example, consider the boundary grid on the right in Figure 8, with the set of vertices (11), (55), (65), (67) with weights $1,2,1,1$. These vertices form an independent set in the down-core of this boundary grid. If we inflate the vertices into increasing sequences with length determined by the weights (making sure every rectangular region inside the grid contains an increasing sequence of points) we reclaim the permutation 
(11)(12)(13)(10)293671845. This same set of vertices is an independent set in the upcore of the same grid. If we instead inflate the vertices into decreasing sequences we obtain the permutation $(11)(12)(13)(10) 297641835$. Finally, these vertices are not an independent set in the updown-core on the grid, since there are edges between the vertices (55), (65), as well as (65), (67).

With these definitions we can state the main result on these new cores:

Proposition 4.7. (1) There is a bijection from the set of permutations avoiding 1324 and pairs $(B, I)$ where $B$ is the boundary grid of a 123-avoiding permutation and $I$ is a weighted independent set in the down-core of $B$. In other words

$$
\operatorname{Av}(1324)=\bigsqcup_{\pi \in \operatorname{Av}(123)} \operatorname{dperms}(\operatorname{bg}(\pi))
$$

(2) Similarly, there is a bijection from the set of permutations avoiding 1234 and pairs $(B, I)$ where $B$ is the boundary grid of a 123-avoiding permutation and $I$ is a weighted independent set in the up-core of $B$. In other words

$$
\operatorname{Av}(1234)=\bigsqcup_{\pi \in \operatorname{Av}(123)} \operatorname{uperms}(\operatorname{bg}(\pi))
$$

(3) Finally, there is a bijection from the set of permutations avoiding 1234 and 1324 and pairs $(B, I)$ where $B$ is the boundary grid of a 123-avoiding permutation and $I$ is an (unweighted) independent set in the updown-core of $B$. In other words

$$
\operatorname{Av}(1234,1324)=\bigsqcup_{\pi \in \operatorname{Av}(123)} \operatorname{udperms}(\operatorname{bg}(\pi))
$$

Proof. We only prove the statement for $\operatorname{Av}(1324)$ as the other statements can be proved by the same arguments. Let $\pi$ be a permutation in $\operatorname{Av}(1324)$. The boundary $\partial(\pi)$ is a 123 -avoiding permutation and we need to prove that the boundary encoding, $\operatorname{benc}(\pi)$, of $\pi$ corresponds to a weighted independent set in the down-core of $\operatorname{bg}(\pi)$. It is clear that the encoding corresponds to a weighted set of vertices in the core. Assume that there is an edge between two of these vertices, say $(i j),(k \ell)$, with $i<k, j<\ell$. By the definition of the down-core this implies that the rectangle $\{i, i+1, \ldots, k\} \times\{j, j+1, \ldots, \ell\}$ is a subset of the core. By the definition of the boundary grid there is at least one left-toright minimum southwest of this rectangle, as well as a right-to-left maximum to the northeast of it. But these two boundary points, together with a point from the box $(i j)$ and a point from the box $(k \ell)$ form an occurrence of 1324 . This is a contradiction, and therefore there cannot be an edge between any of these vertices. This shows that $\operatorname{Av}(1324)$ is a subset of the disjoint union on the right. The other subset relation is similar and left to the reader.

Our main focus will be on subclasses of the first and third permutation classes in the equations above, as their enumerations are unknown. We start by considering the subclass $\operatorname{Av}(1324,2143)$ of $\operatorname{Av}(1324)$. 
4.1. Down-cores and smooth permutations. The smooth permutations are those that correspond to smooth Schubert varieties. Sandhya and Lakshmibai [10] showed that these permutations are the class $\operatorname{Av}(1324,2143)$. The enumeration first appeared in an unpublished preprint of Haiman [9] and is given by the generating function

$$
\frac{1-5 z+3 z^{2}+z^{2} \sqrt{1-4 z}}{1-6 z+8 z^{2}-4 z^{3}} .
$$

Bousquet-Mélou and Butler [4] provided an independent proof of this generating function, and Slofstra and Richmond [11] have found the enumeration of smooth Schubert varieties of all classical finite types, the case of permutations being type $A$. Below we will rederive the generating function (3) while keeping track of the number of boundary points and size of the independent set.

Our approach uses the equation

$$
\operatorname{Av}(1324,2143)=\bigsqcup_{\pi \in \operatorname{Av}(123,2143)} \operatorname{dperms}(\operatorname{bg}(\pi)),
$$

which follows from Proposition 4.7 (1), and the fact that a 1324-avoiding permutation $\pi$ contains 2143 if and only if $\partial(\pi)$ contains 2143 . West [14] proved that the enumeration of the boundaries $\operatorname{Av}(123,2143)$ is given by the alternate Fibonacci numbers [13, A001519]. The following lemma gives a structural description of the boundaries suitable for our purposes.

Definition 4.8. For a boundary grid $B$, we define the reflected grid, $B^{\prime}$, as the grid obtained by reflecting $B$ along a southeast-northwest diagonal.

It follows from Definition 4.3 that $D(B)$ is isomorphic to $D\left(B^{\prime}\right), U(B)$ is isomorphic to $U\left(B^{\prime}\right)$, and $U D(B)$ is isomorphic to $U D\left(B^{\prime}\right)$.

Lemma 4.9. Let $\pi$ be a skew-indecomposable permutation in $\operatorname{Av}(123,2143)$ of length greater than one. The boundary grid of $\pi$ is a union of a sequence of grids, which alternate between staircase grids and reflected staircase grids, which share their extremal boxes; see Figure 11 (left).

Proof. Let $\pi$ be a skew-indecomposable permutation that avoids 123 and 2143 . We assume $\pi$ starts with a descent, i.e., $\pi_{1}>\pi_{2}$. Let $\pi_{1}>\pi_{2}>\cdots>\pi_{s}$ be the initial decreasing sequence of $\pi$, so $\pi_{s+1}>\pi_{s}$ (if $\pi_{s}$ were the last point in $\pi$ then it would be skew-decomposable). If $\pi_{1}>\pi_{s+1}$ then there must be be a point $p$ in $\pi$ to the right of $\pi_{s+1}$ with $p>\pi_{1}$, otherwise $\pi$ is skew-decomposable. Then $\pi_{s} \pi_{s+1} p$ is an occurrence of 123, a contradiction. Therefore $\pi_{s+1}>\pi_{1}$. If $\pi_{s+1}$ is the last point in the permutation the claim holds. If $\pi_{s+1}$ is not the last point in the permutation then the difference in values between $\pi_{i}$ and $\pi_{i+1}$ for $i=1, \ldots, s-2$ must be 1 , for if this failed for a specific $i$ the subsequence $\pi_{i+1} \pi_{i+2} \pi_{s+1} p$ (where $p$ is a point in the permutation with value between $\pi_{i}$ and $\pi_{i+1}$ and index greater than $s+1$ ) is an occurrence of 2143. It follows that rows $1,2, \ldots, s$ in $\operatorname{bg}(\pi)$ have the shape of a staircase grid $B_{s}$. If $\pi_{s-1}-\pi_{s}=1$ then this is the entirety of the boundary grid of $\pi$. Otherwise we consider the decreasing sequence of points northeast of the point $\pi_{s}$. A very similar argument shows that rows $s, s+1, \ldots, s+r$ (where $r+1$ is the length of the decreasing sequence), up to and 
including column $s+r$ have the shape of a reflected staircase grid $B_{r}^{\prime}$. This alternation between staircase grids, and their reflections, persists until the end of the permutation.

The case where $\pi$ starts with an ascent, i.e., $\pi_{1}<\pi_{2}$ can be done with the same argument, the only difference is that the top-most box, which is then alone in the top row, is then the first staircase grid $B_{1}$.

To enumerate the class $\operatorname{Av}(1324,2143)$ we need to enumerate the independent sets of the down-cores on the boundary grids described by the previous lemma. To this end we need to split those grids into smaller grids of the following type: Let $E B_{n}$ be the grid obtained by doubling the top-most column in the staircase grid $B_{n}$, see Figure 10 . Let $E D_{n}$ be the down-core of this grid 2

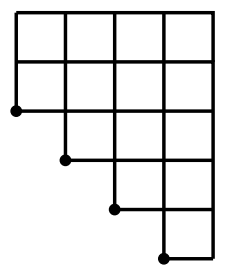

Figure 10. The grid $E B_{4}$.

Lemma 4.10. Let $G=G(x, y)$ be the generating function where the coefficient of $x^{n} y^{k}$ is the number of independent sets of size $k$ in $E D_{n}$. Then

$$
G=\frac{F-1}{(1+y) x}
$$

where $F=F(x, y)$ is the generating function in Theorem 2.4.

Proof. If a box (with an attached left-to-right minimum) is added on the left of the topmost row of $E B_{n}$, then no new edges are added to the core. In fact we obtain the core $B_{n+1}$. Therefore $F=(1+y) x G+1$ and solving for $G$ gives the claimed equation.

With this in hand we can enumerate the independent sets of the cores in Lemma 4.9.

Lemma 4.11. Let $P_{\text {ind }}=P_{\text {ind }}(x, y)$ be the generating function where the coefficient of $x^{n} y^{k}$ is the number of independent sets of size $k$ in down-cores on boundaries of skew-indecomposable permutations from $\operatorname{Av}_{n}(123,2143)$. Then

$$
P_{\text {ind }}=\frac{x(F-1)}{2-G}+x
$$

where $F=F(x, y)$ is the generating function in Theorem 2.4. and $G=G(x, y)$ is the generating function in Lemma 4.10 .

Proof. As in Lemma 4.9 we consider a skew-indecomposable permutation $\pi$ that avoids 123 and 2143. Consider the top-most staircase grid $B_{n}$ described in the proof of Lemma 4.9 (see the grid on the left in Figure 11). We separate this grid from the boundary grid of $\pi$. The top-most point of $\pi$ makes up for the missing left-to-right

\footnotetext{
" $\mathrm{E}$ " is for extended.
} 

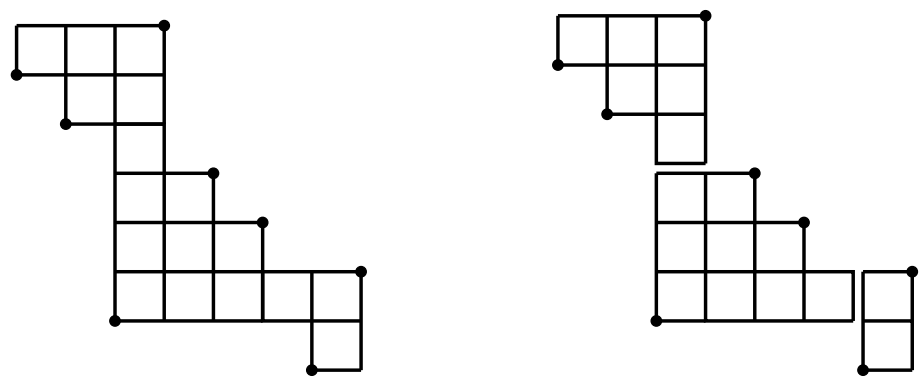

Figure 11. A skew-indecomposable boundary in $\operatorname{Av}(123,2143)$ is shown on the left, and on the right how it is decomposed in the proof of Lemma 4.11.

minimum that should be attached to the bottom-most box of $B_{n}$, see Figure 11 (right). The next piece of the boundary grid is a reflected $E B_{m}$ for some $m \geq 0$, and again the total number of points attached to the grid is what it should be, even though they do not technically appear in the correct place. We keep going and next have another extended boundary grid, and then this alternates between extended boundary grids and their reflections. We proceed in this manner until the boundary grid of $\pi$ is exhausted. Note that there will be one extra boundary point left over.

Since the core of a reflected grid is isomorphic to the core of the unreflected grid we get that the enumeration of independent sets is the same in both. Thus the enumeration of the core on the boundary grid of $\pi$ is

$$
P_{\text {ind }}=(F-1) \cdot \frac{1}{1-(G-1)} \cdot x+x,
$$

where $(F-1)$ enumerates a non-empty staircase grid, 1/(1- $(G-1))$ accounts for the (possibly empty) sequence of extended staircase grids (where every other grid is reflected), and then we multiply by $x$ to track the extra boundary point. We add $x$ for the empty grid, representing the permutation 1.

Proposition 4.12. Let $P=P(x, y)$ be the generating function where the coefficient of $x^{n} y^{k}$ is the number of independent sets of size $k$ in down-cores on boundaries given by a permutation in $\operatorname{Av}_{n}(123,2143)$. Then

$$
P=\frac{1}{1-P_{\text {ind }}} .
$$

By setting $x=z$ and $y=z /(1-z)$ we recover the generating function (3) for the number of $(1324,2143)$-avoiding permutations.

Proof. Since the patterns 1324 and 2143 are skew-indecomposable this follows from Remark 4.5 .

4.2. Updown-cores and the class $\operatorname{Av}(1234,1324,2143)$. The permutation class $\operatorname{Av}(1234,1324)$ is conjectured to have a non- $D$-finite generating function by Albert et. al [1, Section 
6.4]. In this section, we show that the subclass that avoids 2143 has the rational generating function

$$
\frac{1-3 z-2 z^{3}}{1-4 z+2 z^{2}-2 z^{3}+z^{4}}
$$

Similar to the previous section we use the equation

$$
\operatorname{Av}(1234,1324,2143)=\bigsqcup_{\pi \in \operatorname{Av}(123,2143)} \operatorname{udperms}(\operatorname{bg}(\pi)),
$$

which follows from Proposition 4.7 (3). Lemma 4.9 still describes the boundaries of these permutations, and we will need to perform the same type of analysis as in the previous section, with the difference that we need to understand the updown-cores of boundary grids of permutations in $\operatorname{Av}(123,2143)$.

Lemma 4.13. Let $R=R(x, y)$ be the generating function where the coefficient of $x^{n} y^{k}$ is the number of independent sets of size $k$ in updown-cores of $B_{n}$. Then

$$
R=1+x R+\frac{x y R}{1-x} .
$$

Proof. The empty grid $B_{0}$ has one independent set, namely the empty set which gives the first term $x^{0} y^{0}=1$ in the equation above. Assume $n>0$. An independent set in $U D\left(B_{n}\right)$ can contain at most one vertex from the top row. If it contains no vertex then the remaining vertices form a graph isomorphic to $U D\left(B_{n-1}\right)$. This case gives the term $x R$. Otherwise, there is exactly one vertex in the top row, see Figure 12, If the vertex is in box $(1 j)$ then it is connected to every vertex in row $1,2, \ldots, j$, and no other vertices. The induced subgraph on the vertices in the remaining rows is a graph isomorphic to $U D\left(B_{n-j}\right)$. There are $j$ left-to-right minima to the left of the box $(1 j)$. This case gives the last term in the equation: $x /(1-x)$ for the minima to the left of $(1 j), y$ for the box $(1 j)$, and $R$ for the subgraph below.

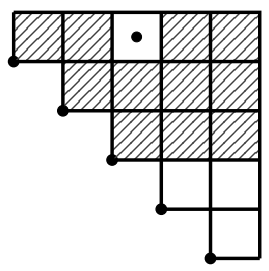

Figure 12. Illustration of the proof of Lemma 4.13

Lemma 4.14. Let $Q_{\text {ind }}=Q_{\text {ind }}(x, y)$ be the generating function where the coefficient of $x^{n} y^{k}$ is the number of independent sets of size $k$ in updown-cores on boundaries of skew-indecomposable permutations in $\operatorname{Av}_{n}(123,2143)$. Then

$$
Q_{\text {ind }}=x+x^{2}+x^{2} y+Q_{\text {up }}+Q_{\text {down }},
$$

where $Q_{u p}=Q_{u p}(x, y), Q_{\text {down }}=Q_{\text {down }}(x, y)$ satisfy the equations

$$
Q_{u p}=x R-x-x^{2}-x^{2} y+\frac{(x R-x) Q_{\text {down }}}{x}+\frac{x^{2} y R\left(Q_{u p}+Q_{\text {down }}+x^{2}+x^{2} y\right)}{1-x},
$$




$$
Q_{\text {down }}=x R-x-x^{2}-x^{2} y+\frac{(x R-x) Q_{u p}}{x}+\frac{x^{2} y R\left(Q_{u p}+Q_{d o w n}+x^{2}+x^{2} y\right)}{1-x},
$$

and $R=R(x, y)$ is given in Lemma 4.13 .

Proof. As before we will use $x$ to track the number of boundary points and $y$ to track the size of independent sets. Recall the description given in Lemma 4.9 of the boundary grid $B$ of a skew-indecomposable permutation $\pi$ in $\operatorname{Av}(123,2143)$. There are three types of grids we distinguish here:

(1) First, when the length of $\pi$ is one or two. Then we can directly see that we get the terms $x+x^{2}(1+y)$ in the equation for $Q_{\text {ind }}$.

(2) Next we assume that $\pi$ starts with a descent so we can separate a staircase grid $B_{b}$ (with $b>1$ ) from the entire grid. Let $Q_{u p}$ to be the generating function enumerating the number of independent sets for this type.

(3) Finally, $\pi$ could start with a ascent. Then we may consider the leftmost part of the boundary grid $B$ as a reflected staircase grid $B_{b}^{\prime}$ (with $b>1$ ). Let $Q_{\text {down }}$ be the generating function enumerating the number of independent sets for this type.

We derive the functional equation satisfied by $Q_{u p}$. If the boundary grid $B$ has only one part in its skew-sum, then it is isomorphic to $B_{b}$. This gives the terms $x R-x-$ $x^{2}-x^{2} y$ in the equation for $Q_{u p}$. Let $B$ be a boundary with at least two parts in its skew-decomposition, where the left-most part is some $B_{b}$ with $b>1$. Let $S$ be the set of vertices $S=\{(i b) \mid i=1, \ldots, b-1\}$, in the last column of $B_{b}$. These vertices are in the same column, so an independent set in $U D(B)$ contains at most one vertex from $S$. We consider two cases.

C1 Assume that the independent set contains no vertex in $S$. If we partition the vertices in $U D(B)$ as the sets $L=\{(i j) \mid i \leq b-1$ and $(i j) \in U D(B)\}$ and $R=\{(i j) \mid i \geq b, j \geq b$ and $(i j) \in U D(B)\}$, then there will be no edges connecting between $L$ and $R$. Moreover, the induced subgraph of $L$ is isomorphic to $U D\left(B_{b-1}\right)$ and the induced subgraph of $R$ is isomorphic to $U D\left(B^{\prime}\right)$ for some boundary grid $B^{\prime}$ in $Q_{\text {down }}$. This gives the $\frac{(x R-x) Q_{\text {down }}}{x}$ term in the functional equation. An example of this case is shown on the left in Figure 13.

C2 Assume that the independent set contains a vertex from $S$, say $b c$, then it can contain no vertices in the set $M=\{(i j) \mid c \leq j \leq b$ and $(i j) \in U D(B)\}$. Partition the remaining vertices into the sets $L=\{(i j) \mid j \leq c$ and $(i j) \in U D(B)\}$ and $R=\{(i j) \mid j>b$ and $(i j) \in U D(B)\}$. There are no edges between $L$ and $R$, and moreover, the induced subgraph of $L$ is isomorphic to $U D\left(B_{c-1}\right)$, and the induced subgraph of $R$ is isomorphic to $U D(B)$ for some boundary grid $B$ of a skew-indecomposable permutation in $\operatorname{Av}(123,2143)$. This implies the $\frac{x^{2} y R\left(Q_{u p}+Q_{\text {down }}+x^{2}+x^{2} y\right)}{1-x}$ term in the functional equation, where the $1 /(1-x)$ accounts for the points attached to the boxes corresponding to the vertices in $M$. An example of this case is shown on the right in Figure 13 .

The justification for the equation given for $Q_{\text {down }}$ is a symmetry of the arguments given for $Q_{u p}$. 

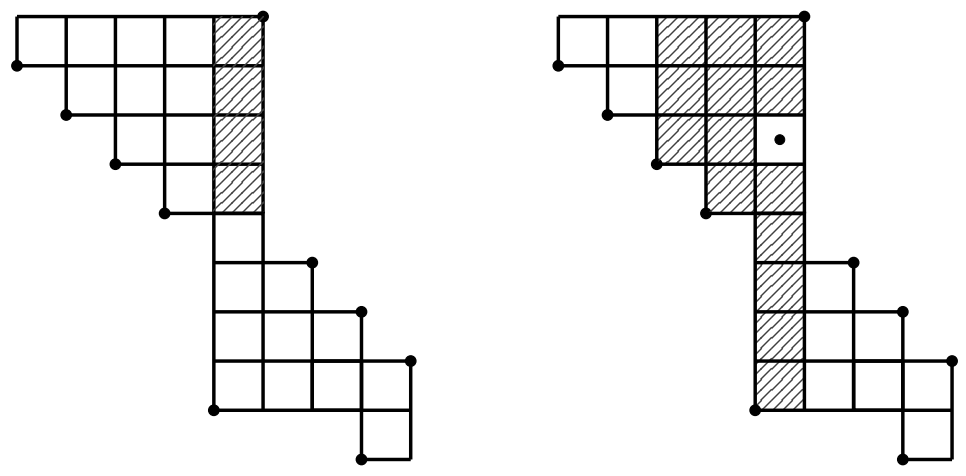

Figure 13. On the left is an example of case $\mathrm{C} 1$ in the proof of Lemma 4.14, and on the right an example of case $\mathrm{C} 2$.

Remark4.5implies we can find the generating function for $(1234,1324,2143)$-avoiding permutations.

Proposition 4.15. Let $Q=Q(x, y)$ be the generating function where the coefficient of $x^{n} y^{k}$ is the number of independent sets of size $k$ in updown-cores on boundaries from $\operatorname{Av}_{n}(123,2143)$. Then

$$
Q=\frac{1}{1-Q_{\text {ind }}} .
$$

By setting $x=z$ and $y=z$ we get the generating function (5) for the number of (1234, 1324, 2143)-avoiding permutations.

The generating function gives the enumeration

$$
1,1,2,6,21,75,268,958,3425,12245,43778,156514,559565, \ldots
$$

and has been added to the Online Encyclopedia of Integer Sequences [13, A263790].

4.3. Updown-cores and the class $\operatorname{Av}(1234,1324,1432,3214)$. Here we replace avoidance of 2143 with avoidance of 1432 and 3214 and show that this subclass has the rational generating function

$$
\frac{1-z-z^{2}-z^{3}}{1-2 z-z^{2}-2 z^{3}-4 z^{4}-8 z^{5}+15 z^{7}+14 z^{8}+7 z^{9}} .
$$

If a permutation $\pi$ avoids 1324, then it contains 1432 (3214) if and only if $\partial(\pi)$ contains 1432 (3214). Proposition 4.7 (3) therefore implies the equation

$$
\operatorname{Av}(1234,1324,1432,3214)=\bigsqcup_{\pi \in \operatorname{Av}(123,1432,3214)} \operatorname{udperms}(\operatorname{bg}(\pi)) .
$$

As before we need a structural description of the boundary grids indexed in the disjoint union above:

Lemma 4.16. A boundary grid of a skew-indecomposable permutation in

$$
\operatorname{Av}(123,1432,3214) \backslash\{1,12,2143\}
$$


is an alternating sequence of grids of the form $\mathrm{bg}(132)$ and $\mathrm{bg}(213)$ sharing their northwestern-most box with the southeastern-most box of the next grid.

Proof. Let $\pi$ be a skew-indecomposable permutation in $\operatorname{Av}(123,1432,3214) \backslash\{1,12,2143\}$. The number of points northeast of a left-to-right minimum in $\pi$ is at most two, and likewise the number of points southwest of a right-to-left maximum in $\pi$ is at most two. We leave it to the reader to show that the boundary grid of $\pi$ has the claimed structure by following a similar argument as in the proof of Lemma 4.14. See Figure 14.

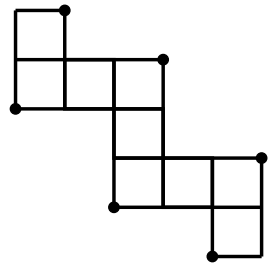

FIGURE 14. The boundary grid of the permutation 462513.

Having the structural description of the boundary grids allows us to enumerate the updown-cores by the size of the independent set.

Lemma 4.17. Let $S_{\text {ind }}=S_{\text {ind }}(x, y)$ be the generating function where the coefficient of $x^{n} y^{k}$ is the number of independent sets of size $k$ in updown-cores on boundaries of skew-indecomposable permutations in $\operatorname{Av}_{n}(123,1432,3214)$. Then

$$
S_{\text {ind }}=x+x^{2}(1+y)+x^{4}\left(1+7 y+7 y^{2}\right)+S_{u p}+S_{\text {down }},
$$

where $S_{\text {up }}=S_{\text {up }}(x, y), S_{\text {down }}=S_{\text {down }}(x, y)$ satisfy the equations

$$
\begin{aligned}
& S_{\text {up }}=x^{3}\left(1+3 y+y^{2}\right)+x S_{\text {down }}+x y S_{\text {down }}+x^{2} y\left(S_{\text {up }}+(1+y) x^{2}\right), \\
& S_{\text {down }}=x^{3}\left(1+3 y+y^{2}\right)+x S_{\text {up }}+x y S_{\text {up }}+x^{2} y\left(S_{\text {down }}+(1+y) x^{2}\right) .
\end{aligned}
$$

Proof. We define $S_{u p}$ to be the generating function for the skew-indecomposable grids whose right-most grid is of the form $\operatorname{bg}(213)$ and $S_{\text {down }}$ for the skew-indecomposable grids whose right-most grid is of the form $\mathrm{bg}(132)$. Therefore if either has only one part it will be counted by $x^{3}\left(1+3 y+y^{2}\right)$. The derivation of the equations for $S_{u p}$ and $S_{\text {down }}$ are analogous to the proof of Lemma 4.14 and are omitted. We add in $x$ for the single point, $x^{2}(1+y)$ for $\operatorname{bg}(12)$ and $x^{4}\left(1+7 y+7 y^{2}\right)$ for $\operatorname{bg}(2143)$.

Remark 4.5 implies we can find the generating function for $(1234,1324,1432,3214)$ avoiding permutations.

Proposition 4.18. Let $S=S(x, y)$ be the generating function where the coefficient of $x^{n} y^{k}$ is the number of independent sets of size $k$ in updown-cores on boundaries from $\operatorname{Av}_{n}(123,1432,3214)$. Then

$$
S=\frac{1}{1-S_{\text {ind }}} .
$$

By setting both $x=z$ and $y=z$ we get the generating function (6) for the number of (1234, 1324, 1432, 3214)-avoiding permutations. 
The generating function gives the enumeration

$$
1,1,2,6,20,62,172,471,1337,3846,11030,31442,89470,254934 \ldots
$$

and has been added to the Online Encyclopedia of Integer Sequences [13, A260696].

\section{Conclusions and future Work}

Growth rates. As already noted the enumeration for the number of permutations that avoid 1324 is unknown, and not even the growth rate, i.e., the limit

$$
\lim _{n \rightarrow \infty} \sqrt[n]{\operatorname{Av}_{n}(1324)}
$$

is known. Recently Bevan et al. [3] established that the growth rate is in the interval $[10.271,13.5]$. We hope that the methods developed here can lead to some non-trivial lower bounds for this growth rate, even though the classes studied here did not yield good bounds, see Table 3 .

\begin{tabular}{llc} 
Class & Reference & $\approx$ growth rt. \\
\hline $\operatorname{Av}(1324,2143)$ & Prop. 4.12, eqn (31) & 4.4 \\
$\operatorname{Av}(1234,1324,2143)$ & Prop. 4.15, eqn. (55) & 3.6 \\
$\operatorname{Av}(1234 ; 1324 ; 1432 ; 3214)$ & Prop. 4.18, eqn. (6) & 2.9
\end{tabular}

TABLE 3. The growth rates for the subclasses of $\operatorname{Av}(1324)$ studied in this paper.

Generalized patterns. We have focused on classical pattern avoidance in this paper. It is likely that our methods can be used to study the avoidance of generalized patterns. For example consider the sets

$$
\begin{aligned}
& \operatorname{Av}(1 \underline{23} 4)=\{\pi \mid \pi \text { avoids an occurrence of } 1234 \text { with } 2 \text { and } 3 \text { adjacent }\} \\
& \operatorname{Av}(1 \underline{32} 4)=\{\pi \mid \pi \text { avoids an occurrence of } 1324 \text { with } 3 \text { and } 2 \text { adjacent }\},
\end{aligned}
$$

which are defined by vincular patterns, see Babson and Steingrimsson [2]. Our methods, give a bijection between the two sets, by first mapping to the boundary encoding, and then back to permutations by reversing the points in every column.

Non-crossing and non-nesting partitions. It was noted by Galashin [7] that bumpdiagrams, see e.g., Rubey and Stump [12], for non-crossing and non-nesting partitions, provide an alternative framework for the independent sets in 132- and 123-cores.

From empirical testing, it seems that the down-cores on boundaries avoiding 123 are pure if and only if the boundary avoids 2143. We record this as a conjecture:

Conjecture 5.1. Let $\pi$ be a 123-avoiding permutation. The independent set complex of the down-core $D(\operatorname{bg}(\pi))$ is pure if and only if $\pi$ avoids 2143 .

Acknowledgements. We thank Émile Nadeau and the anonymous referees for thoughtful comments that helped with clarifying the presentation of these results. 


\section{REFERENCES}

[1] M. Albert, C. Homberger, J. Pantone, N. Shar, and V. Vatter, Generating permutations with restricted containers, Journal of Combinatorial Theory, Series A 157 (2018), 205 -232.

[2] E. Babson and E. Steingrimsson, Generalized permutation patterns and a classification of the Mahonian statistics, Sém Lothar Combin (2000).

[3] D. Bevan, R. Brignall, A. Price, and J. Pantone, Staircases, dominoes, and the growth rate of 1324avoiders, Electronic Notes in Discrete Mathematics 61 (2017), 123 -129. The European Conference on Combinatorics, Graph Theory and Applications (EUROCOMB'17).

[4] M. Bousquet-Mélou and S. Butler, Forest-like permutations, Ann. Comb. 11 (2007), no. 3-4, 335354.

[5] L. Comtet, Advanced combinatorics, enlarged, D. Reidel Publishing Co., Dordrecht, 1974. The art of finite and infinite expansions.

[6] P. Flajolet and M. Noy, Analytic combinatorics of non-crossing configurations, Discrete Math. 204 (1999), no. 1-3, 203-229.

[7] P. Galashin. Personal communication.

[8] I. Gessel, Symmetric Functions and P-Recursiveness, Journal of Combinatorial Theory, Series A 53 (March 1990), no. 2, 257-285.

[9] Mark Haiman, Enumeration of smooth Schubert varieties, 1992. unpublished.

[10] V. Lakshmibai and B. Sandhya, Criterion for smoothness of Schubert varieties in $\operatorname{Sl}(n) / B$, Proc. Indian Acad. Sci. Math. Sci. 100 (1990), no. 1, 45-52.

[11] E. Richmond and W. Slofstra, Staircase diagrams and enumeration of smooth Schubert varieties, Journal of Combinatorial Theory, Series A 150 (2017), 328 -376.

[12] M. Rubey and C. Stump, Crossings and nestings in set partitions of classical types, Electron. J. Combin. 17 (2010), no. 1, Research Paper 120, 19.

[13] N. Sloane, The on-line encyclopedia of integer sequences. published electronically at http://oeis.org, 2010.

[14] J. West, Generating trees and forbidden subsequences, Discrete Mathematics 157 (1996), no. 1-3, 363-374.

School of Computer Science, Reykjavik University, Reykjavik, Iceland

E-mail address: christianbean@ru.is

School of Computer Science, Reykjavik University, Reykjavik, Iceland

E-mail address: murray140ru.is

School of Computer Science, Reykjavik University, Reykjavik, Iceland

E-mail address: henningu@ru.is 